\title{
ANÁLISIS DE ACTITUDES POSITIVAS Y NEGATIVAS HACIA EL HABLA COSTARRICENSE
}

\author{
Annette Calvo Shadid*
}

\begin{abstract}
RESUMEN
Estudio sociolingüístico sobre actitudes lingüísticas positivas y negativas hacia el español de Costa Rica, suministradas por hablantes de cuatro distritos de la ciudad capital. Se trata de dos preguntas abiertas: ¿En cuáles zonas del país considera usted que hablan mejor? y ¿En cuáles zonas del país considera usted que hablan peor? y el porqué de su respuesta. Se establecen categorías de acuerdo con las repuestas suministradas, se corrobora con qué variedad del español de Costa Rica se identifican estos hablantes, se describe cómo evalúan las otras variedades lingüísticas, y se analiza con cuáles ideologías sociales subyacen a sus actitudes lingüísticas.

Palabras clave: Español de Costa Rica, sociolingüística, actitudes lingüísticas, discriminación lingüística, prestigio lingüístico.
\end{abstract}

\begin{abstract}
This is a sociolinguistic study of positive and negative attitudes toward Costa Rican Spanish. The people whose views were considered are Spanish speakers from four different districts of the capital city of San Jose. They were asked to respond to two open questions: Where in Costa Rica do you believe people speak the best? and Where do you believe they speak the worst? They were then asked to explain their responses. The author then categorizes the answers given, verifies the variety of Costa Rican Spanish spoken by the respondents, describes the ways in which they evaluate varieties distinct from their own, and analyses the social ideologies that underlie their attitudes.
\end{abstract}

Key Words: Costa Rican Spanish, Sociolinguistics, linguistic attitudes, linguistic discrimination, linguistic prestige.

Universidad de Costa Rica. Decana, Facultad de Letras. Costa Rica.

Correo electrónico: acalsha@yahoo.com.mx

Recepción:26/02/14. Aceptación: 07/04/14. 


\section{Introducción}

Como parte de un estudio sociolingüístico sobre las actitudes lingüísticas de los costarricenses hacia el español ${ }^{1}$ (hablantes de la ciudad capital de San José), se tomaron en cuenta las actitudes positivas y negativas de los hablantes acerca de las diferentes variedades del español de Costa Rica.

Por las características del estudio, las preguntas abiertas sobre por qué consideraban mejores o peores las variedades de habla dentro del país (actitudes positivas y negativas) no fueron analizadas a profundidad en aquel momento. La razón de esto fue que, sin contar las preguntas abiertas, el documento total constaba de 40 preguntas, las cuales se debieron procesar, tabular y analizar. En su oportunidad se elaboraron categorías generales para cada tipo de respuesta, con el fin de clasificar, grosso modo, las respuestas a las preguntas abiertas, pero no se llevó a cabo un análisis de ellas.

En el presente estudio se analizarán tales actitudes, con el fin de determinar categorías más detalladas y corroborar, mediante las respuestas, con qué variedad del español de Costa Rica se identifican los hablantes, cómo evalúan las distintas variedades del español de Costa Rica, y con qué ideologías y estereotipos sociales (prestigio, discriminación) se relacionan sus actitudes lingüísticas.

\section{Antecedentes}

Además de los trabajos que teorizan acerca de las actitudes lingüísticas como los de Quesada Pacheco (2005 y 2008) y los de Umaña Aguiar (1989 y 1990), a continuación se exponen con más detalle estudios sobre el campo. En Arrieta, Jara y Pendones (1986/2010²) se describen las actitudes lingüísticas de una muestra de estudiantes de secundaria con respecto a dos variedades de habla que se distinguen claramente en Costa Rica: el habla del Valle Central y el habla de Guanacaste. El estudio resulta congruente con la propuesta de que las actitudes están relacionadas con dos aspectos: la inseguridad y los prejuicios lingüísticos.

El análisis de los datos comprobó que, en general, todos los estudiantes calificaron más positivamente a los hablantes del Valle Central que a los de Guanacaste en todos los rubros. Es importante el resultado de los estudiantes del colegio que representaba la clase media baja de Guanacaste, pues la diferencia de calificación resultó significativa en todos los ejes, por lo cual las autoras señalan la particular inseguridad lingüística de estos hablantes.

Jaén García (1991) presenta una tesis sobre las actitudes lingüísticas de los hablantes de Sardinal, Carrillo, Guanacaste, acerca de su propia habla. Se trata de un estudio local que muestra un alto grado de identificación con su habla y la población pareciera estar identificada con los valores de la comunidad.

Solano Rojas y Umaña Aguiar (1994) presentan una investigación sobre la inseguridad lingüística de los estudiantes universitarios costarricenses respecto del español. Los estudiantes consideraron, en su gran mayoría, que los costarricenses no hablan bien el español, especialmente en cuanto al léxico, que es el campo, según las autoras (1994:173), “en que más fácilmente se verbaliza la inseguridad". De este, lo correspondiente a "tipo de vocabulario usado", como "pobre/ escaso/ simple/ repetitivo", incluyendo los "costarriqueñismos" (1994:173), muestra los porcentajes mayores de por qué piensan los estudiantes que se habla mal, lo cual refleja desprecio por lo costarricense, lo "propio". En cuanto a la pronunciación, los rasgos más frecuentes fueron "la mala/pésima/ marcada pronunciación" y la "pronunciación de 'r' y 'rr'" (1994:174). Respecto de otros factores, el de la presión de grupo fue el más marcado, y luego de este, la variación pronominal (ustedeo, voseo, tuteo).

Las autoras concluyen (1994:176) que uno de los factores más importantes de las opiniones negativas respecto del habla costarricense, es que "Los escolarizados están a menudo muy convencidos, gracias a la autoridad del maestro o profesor, de que 'hay cosas que decimos mal o muy mal'. Esto no tendría un efecto tan negativo 
si a la vez se lograra inculcar un verdadero aprecio por el habla costarricense".

El libro de Jara Murillo sobre el español de Costa Rica según los ticos (2006:xi), "es un estudio sobre opiniones acerca del habla de la comunidad propia."

La autora (2006:9) fundamenta su estudio en el campo de la lingüística popular, y lo considera "un campo virgen dentro del área de estudio de la lingüística en nuestro medio e incluso en Latinoamérica." Además, aborda (2006:21) la relación que existe entre las actitudes y el área de la lingüística popular, en tanto las actitudes se relacionan con "cómo la gente siente y percibe lo que se dice", y la lingüística popular, "lo que la gente piensa respecto de lo que se dice". Así, las relaciona (2006:23) como "un continuo, y asignamos al campo específico de la lingüística popular las reacciones más conscientes, discursivamente elaboradas y explícitas, [...]".

Jara Murillo trabajó con 930 entrevistas sobre la base de la pregunta: “¿Qué opina usted sobre el español tal como se habla en Costa Rica?" En general, en todos los niveles de la lengua, a saber, el fonético, el morfosintáctico y el léxico, presentan valoraciones generales bastante negativas. Respecto de la prominencia que todos los aspectos se incluyen en la representación mental del español de Costa Rica, la mayor prominencia se refiere, al igual que el estudio anterior, al vocabulario (34\%). Luego, la influencia del inglés y el uso de extranjerismos (29\%); luego, pareciera que con mucha menos prominencia, el componente de la variación lectal (18\%); con un porcentaje similar, el componente fonético (14\%), para finalizar con el morfosintáctico, que presenta en realidad una muy baja prominencia (5\%).

Sobre los modelos culturales del español de Costa Rica, se percibieron diversas posiciones, por lo que la autora propone cuatro modelos en un continuo de actitudes. Estos se encuentran en un macromodelo compuesto por una actitud neutral, no valorativa en un extremo, una actitud crítica pero modelada en el centro, y las reacciones más actitudinales en el otro extremo, ya sean negativas, por un lado, o positivas, por el otro. Es preciso señalar que la actitud crítica extrema en sentido negativo, presenta el porcentaje más alto (37\%).

Los resultados finales demuestran, según la investigadora, (2006:150) que los costarricenses "padecen" de inseguridad lingüística, lo cual se refleja en las actitudes de que el espCR es un español degradado del "verdadero español" que es el de España o "el Español". La autora finaliza señalando (2006:153) que existe una "actitud policial" con respecto a la lengua, que desde los grupos normativos se ha transferido a los hablantes, lo cual nos hace pensar que nuestra habla "tiene defectos terribles". Sin embargo, reconoce (2006:154) que "la percepción del cambio lingüístico y la variabilidad de la lengua nos deba hacer conceder, en ocasiones, que nuestro español no es ni mejor ni peor que cualquier otro español ni que cualquier otra lengua".

Por su parte, Drevdal (2009) describe las actitudes hacia su propia variedad de un grupo de nicaragüenses que vive en Costa Rica. Toma en cuenta su calidad de inmigrantes y cómo las actitudes pueden ser influenciadas por la experiencia migratoria.

Se seleccionaron 60 sujetos residentes tanto en zonas rurales como urbanas, y tres grupos de edad, para responder un cuestionario. Los principales resultados son los siguientes (Drevdal, 2009:101-104).

Los nicaragüenses perciben una gran diferencia entre el dialecto propio y el habla costarricense; evidencian ser muy conscientes de que otras personas los identifican como nicaragüenses a partir de su manera de hablar. Muestran tener expectativas negativas acerca de la reacción de los costarricenses frente al dialecto nicaragüense, y califican la propia habla de manera bastante negativa en lo que se refiere al prestigio y la movilidad social. Consideran necesario cambiar la manera de hablar para mejorar sus posibilidades en el mercado del trabajo; la gran mayoría de los informantes se muestra renuente a cambiar permanentemente la manera de hablar. Se da importancia al mantenimiento del dialecto nicaragüense en relaciones intragrupales. El rechazo al dialecto 
costarricense es particularmente fuerte en la comunicación con otros nicaragüenses, lo que se puede interpretar como indicador de que el habla juega un papel importante como marca de identidad grupal. Muchos de los encuestados reconocen la necesidad de acomodarse lingüísticamente a los costarricenses por razones instrumentales, es decir, para mejorar su posición social en el país. A partir de estos datos, se puede esperar que exista un cierto nivel de bidialectalismo. Los encuestados que viven en zonas rurales muestran actitudes más negativas que los nicaragüenses residentes en San José.

En resumen, se observa en la mayor parte de los antecedentes que los costarricenses manifiestan inseguridad y prejuicios lingüísticos, y cierta discriminación lingüística en lo que respecta al habla de la periferia del país, por ejemplo, en los casos estudiados, respecto del habla de Guanacaste. A continuación, en el presente trabajo, se repiten muchas de las mismas actitudes que se han dado como resultado en los estudios anteriores: inseguridad, discriminación y prejuicios lingüísticos, entre otros aspectos que manifiestan las actitudes positivas y negativas hacia el habla nacional.

\section{Antecedentes teóricos}

Las actitudes se refieren a juicios de valor hacia las lenguas que tienen que ver, más que con diferencias lingüísticas, con convenciones sociales como las de estatus o privilegio, y con los grupos de poder que las promueven. Las actitudes responden a la percepción, los valores y los estereotipos de los hablantes respecto de la lengua. Los estudios sobre actitudes revelan prejuicios negativos o positivos hacia las comunidad de habla. El prestigio de una variedad siempre está acompañado de un nivel socioeconómico alto y de la variedad usada en una zona focal. Los investigadores de IULA $^{3}$ señalan:

Una lengua vale lo que vale el grupo que la habla. Una lengua tiene poder de atracción si los individuos que la hablan tienen poder adquisitivo, están bien situados socialmente y tienen capacidad de influir en la sociedad. Si se renuncia a hablar una lengua es porque se asocia a los grupos sociales más desfavorecidos, al retraso socioeconómico y a un nivel educativo bajo. http://www.iula.upf.edu/ uval/upres2es.htm.

Sobre el valor de una lengua, López Morales (2004:287) señala las hipótesis planteadas por Giles (1979), la del valor inherente y la de la norma impuesta:

[...] La primera se basa en el juicio de valor que se produce al comparar dos variedades entre sí, independientemente de los hablantes que las manejen; la segunda, apunta a la valoración positiva de una variedad sobre la otra, debido a que es hablada por un grupo de mayor prestigio. Las investigaciones del propio Giles confirman la existencia de la segunda, lo que explica, entre otras cosas, que una misma variedad (o fenómenos caracterizadores de ella) sea valorada de diferente forma, llegando a producir actitudes positivas y negativas, según el grupo social a la que asocie (Edwards, 1982).

Por otra parte, el análisis de las actitudes hacia las lenguas se ha estudiado desde diferentes enfoques, principalmente desde las posiciones conductista o mentalista. Según López Morales (2004:287):

Las caracterizaciones mentalistas lo definen como 'un estado de disposición' (Allport, 1967), 'una variable que interviene entre un estímulo que afecta a la persona y su respuesta a él' (Agheyisi y Fishman, 1970: 138; Cooper y Fishman, 1974: 7). Desde este punto de vista, la actitud de una persona (y, en general, de un grupo determinado) lo prepara para reaccionar de manera específica ante un estímulo dado.

Desde esta concepción, la actitud lingüística no se puede observar o analizar directamente.

Por el contrario, las posiciones conductistas (López Morales 2004:287:288):

[...], basadas en las respuestas que los hablantes dan a ciertas situaciones sociales, pueden estudiarse directamente, sin necesidad de acudir a informes introspectivos individuales, no siempre aprovechables para la investigación. Sin embargo, estas últimas tienen el grave inconveniente científico de que no predicen la conducta verbal (ni ninguna otra) como las mentalistas y, por lo tanto, no pueden constituirse en patrones sistemáticos y coherentes. 
Para el autor (2004:290), la actitud es diferente de la creencia, puesto que aquélla solo puede ser negativa o positiva:

A diferencia de casi todos y a semejanza de Fishbein, separo el concepto de creencia del de actitud, que es, junto al saber proporcionado por la conciencia lingüística, el que las produce. Las actitudes solo pueden ser positivas, de aceptación, o negativas, de rechazo; una actitud neutra es imposible de imaginar (pensando en su naturaleza conativa): se trata más bien de ausencia de actitud.

Por otro lado, van Dijk (1996:19) ha señalado que las actitudes y las opiniones están ligadas a las ideologías, las cuales son sistemas que sustentan las cogniciones sociopolíticas de los grupos:

"[...] las ideologías organizan las actitudes de los grupos sociales que consisten en opiniones generales organizadas esquemáticamente acerca de temas sociales relevantes." Es decir, que también las actitudes y las opiniones acerca de la lengua y los usuarios están organizadas por estos sistemas. Se basan en los valores del grupo. Asimismo, señala (1996:31) que cuando se trata de postular significados en el discurso de un grupo, cuando la dominación no está cuestionada, "las estructuras ideológicas estarán simplemente presupuestas, o bien se pueden considerar como propias del sentido común. En tal caso, se requiere analizar los significados ideológicos haciendo explícitos los significados implícitos y aquellos significados que se toman habitualmente como lugares comunes".

Ante estos antecedentes teóricos, se asume que las actitudes lingüísticas tienen que ver con el valor inherente y la imposición de la norma de una variedad por encima de otra(s), por ser hablada por un grupo de mayor prestigio; en este caso se enfocan desde un punto de vista mentalista, en el sentido en que se expresó más arriba con López Morales (2004), y en el que lo anota Solís (2002:1):

Pel que fa a l'estudi sobre les actituds lingüístiques s'han desenvolupat diferents teories, de les quals, principalment, sobresurten dos: la mentalista i la behaviorista, segons el seu concepte d'actitud. Mentre que el corrent mentalista propugna les actituds lingüístiques com a estats mentals i neurals de disposició (Allport, 1967), no directament observables, però inferibles gràcies a uns estímuls apropiats, el corrent behaviorista considera les actituds com a conductes o respostes a una situació determinada (Agheyisi\&Fishman, 1970).

En este caso, las actitudes se han inferido mediante las percepciones cognitivo-lingüísticas de los hablantes entrevistados. Por otro lado, tales actitudes se ligan con ideologías, instrumentos por medio de los cuales se interpreta el mundo y se actúa en él. Todo ello se contemplará en el análisis de los resultados del presente estudio.

\section{Antecedentes metodológicos}

La población de estudio fueron personas adultas a partir de 20 años de edad, habitantes del cantón Central de San José, ciudad capital. La muestra seleccionada se estableció en 404 entrevistas distribuidas proporcionalmente según la edad, el sexo, el estrato socioeconómico y el nivel educativo, en personas de cuatro distritos ubicados en las diferentes zonas del cantón Central de San José. Para determinar las personas dentro de los distritos se seleccionó una muestra de segmentos censales proporcional a las características del estrato socioeconómico del distrito, definidas por el INEC (Instituto Nacional de Estadística y Censos).

Se seleccionaron aleatoriamente los distritos de San Francisco de Dos Ríos, La Uruca, Pavas y Hatillo, todos correspondientes al cantón central de San José.

La muestra se distribuyó proporcionalmente entre los cuatro distritos seleccionados, con el fin de obtener una muestra autoponderada, y fue aplicada en agosto de 2010.

La encuesta, de 40 ítems, fue distribuida en cuatro partes. La primera parte trató sobre preguntas de actitudes y percepciones del español nacional. La segunda parte versó sobre opiniones acerca de la corrección lingüística y los medios de comunicación, y sobre la unidad o diversidad del español. La tercera parte se refirió a percepciones cognitivo-lingüísticas sobre el español de los otros países, y a actitudes afectivas y preferencias por otras variedades 
lingüísticas: La cuarta parte correspondió a información sociodemográfica.

\section{Descripción y análisis de los resultados}

La parte de la entrevista que aquí se estudia, corresponde solamente a dos preguntas relacionadas con percepciones cognitivo- lingüísticas acerca del español nacional; específicamente, actitudes positivas y negativas. A continuación se presentan los principales datos sociolingüísticos.

\subsection{Actitudes positivas}

¿En cuáles zonas del país considera usted que hablan mejor?

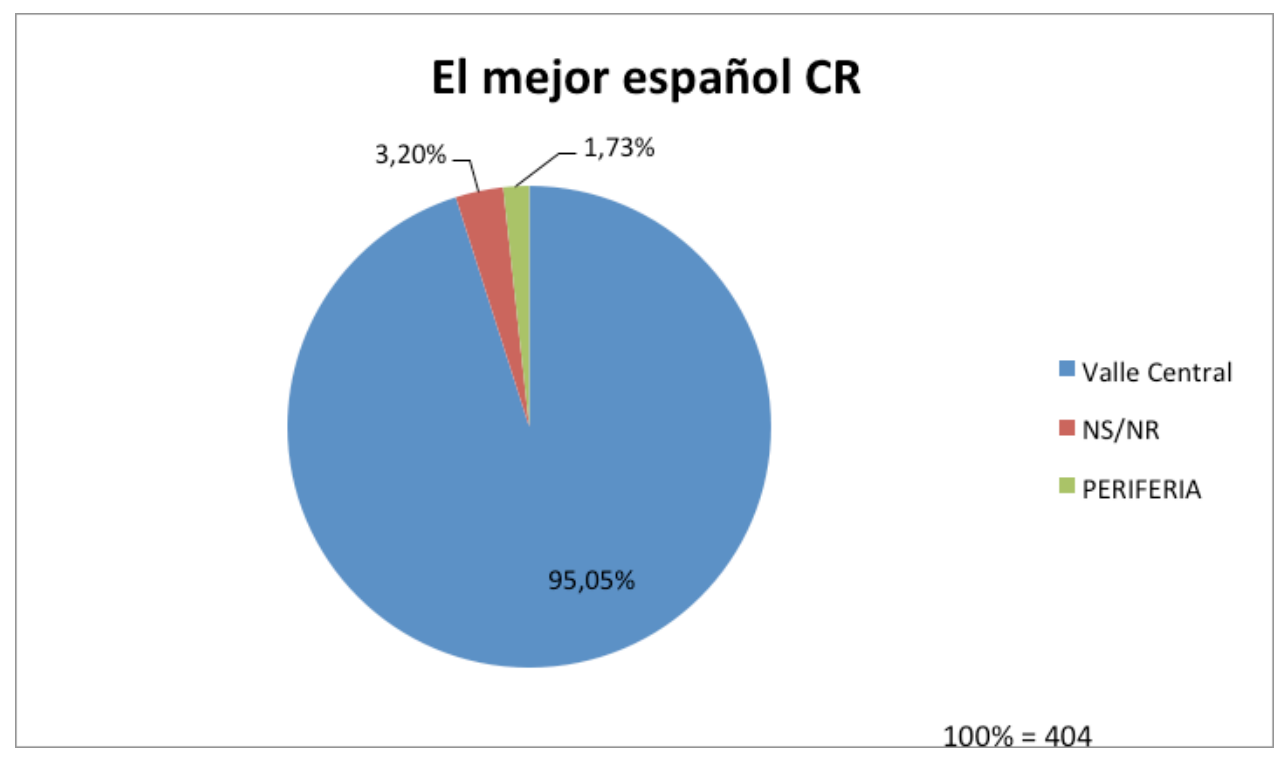

Según el Gráfico 1, el 95\% de los entrevistados indicó que donde se habla mejor es en el Valle Central; se favorece San José (Área Metropolitana) (72\%), seguido por Heredia (10\%). Esto era de esperar pues se sabe que una comunidad de habla recibirá criterios valorativos semejantes sobre una variedad si se trata especialmente de la que se habla en la capital o en una ciudad de las principales de un país. En las respuestas, el mejor español del país se suele dirigir hacia los usuarios, y se supone que es por su manera de hablar, pero también se verá que se relaciona con sus costumbres, su educación, su conducta, su procedencia etc.

Desde décadas atrás se atestigua lo anterior por parte de los investigadores, por opiniones de hablantes de otras regiones del país; véase, por ejemplo, Arrieta, Jara y Pendones (1986:122): "Los datos comprueban que todos los colegios calificaron con valores más positivos a los hablantes del Valle Central que a los de la región de Guanacaste en todos los rubros."

Las opiniones por las cuales a los entrevistados les gusta más el español del Valle Central se detallan abajo. Se establecieron dos tipos de categorías de respuestas: lingüísticas propiamente dichas y sociolingüísticas, cada una con sus respectivas opiniones. A continuación se observan, en las Tablas 1 y 2, todas las categorías en su orden de importancia. 
TABLA 1

Categorías sociolingüísticas: educación y cultura, corrección y prestigio,

\begin{tabular}{|c|c|c|}
\hline CATEGORÍAS & OPINIONES & PORCENTAJES \\
\hline \multirow[t]{3}{*}{ Educación y cultura } & $\begin{array}{l}\text { Buenos estudios, mejor educación, } \\
\text { más facilidades para educarse }\end{array}$ & $87,24 \%$ \\
\hline & Más cultura, más ambiente cultural & $10,70 \%$ \\
\hline & $\begin{array}{l}\text { Las personas son más respetuosas, } \\
\text { más actualizadas, decentes, sueltas, } \\
\text { tranquilas }\end{array}$ & $2,06 \%$ \\
\hline TOTAL & & $100 \%=243$ \\
\hline \multirow[t]{3}{*}{ Corrección y prestigio } & $\begin{array}{l}\text { Se comprende bien o mejor, se } \\
\text { habla con más claridad }\end{array}$ & $64,10 \%$ \\
\hline & $\begin{array}{l}\text { Se expresan bien o mejor, más } \\
\text { correctamente, tienen buen uso del } \\
\text { lenguaje, de la gramática, hablan } \\
\text { con más fluidez }\end{array}$ & 20,51 \\
\hline & $\begin{array}{l}\text { Hablan igual (homogéneo), normal, } \\
\text { sin dichos, un habla más universal, } \\
\text { con más antigüedad }\end{array}$ & $15,30 \%$ \\
\hline TOTAL & & $100 \%=39$ \\
\hline \multirow[t]{3}{*}{ zona central del país } & $\begin{array}{l}\text { Mejor habla en la capital, en las } \\
\text { zonas urbanas, por ser el centro } \\
\text { del país }\end{array}$ & $52,17 \%$ \\
\hline & Mejor comunicación, más contactos & $26,09 \%$ \\
\hline & $\begin{array}{l}\text { Más oportunidades, mayores } \\
\text { facilidades }\end{array}$ & $21,73 \%$ \\
\hline TOTAL & & $100 \%=23$ \\
\hline
\end{tabular}

En términos generales, las categorías sociolingüísticas presentan una mayor proporción de respuestas positivas; esto es muy importante puesto que la lengua no se concibe solamente como un código con una estructura interna determinada, sino que participa también de toda la dinámica social, política y cultural de las comunidades que la usan. Precisamente por la estrecha relación entre la lengua, la sociedad, la cultura y la política, se crean todo tipo de actitudes sobre el valor de una variedad dentro de esta dinámica. En ese sentido, se citan las palabras de Moreno Fernández 4 (2005: 178):
[...] la actitud ante la lengua y su uso se convierte en especialmente atractiva cuando se aprecia en su justa magnitud el hecho de que las lenguas no son solo portadoras de unas formas y unos atributos lingüísticos determinados, sino que también son capaces de transmitir significados o connotaciones sociales, además de valores sentimentales. Las normas y marcas culturales de un grupo se transmiten o enfatizan por medio de la lengua.

Por otro lado, se recuerdan también de nuevo las llamadas "hipótesis del valor inherente y de la norma impuesta" de Giles, 1979 (Citadas por López Morales, 2004:287) que se citaron arriba, sobre todo la segunda, que apunta a la 
valoración positiva de una variedad sobre la otra debido a que es hablada por un grupo de mayor prestigio, se ve reflejada claramente en este caso: la variedad del Valle Central es mejor por ser hablada por gente más educada, con mayor cultura, por personas más decentes y respetuosas, y por ser personas con más contactos, y porque son estas quienes hablan con mayor claridad y fluidez. Además, la del valor inherente también se refleja en las opiniones de forma independiente de los hablantes: la norma proviene de la ciudad capital, del centro del país, de las zonas urbanas, pues es donde se habla con mayor homogeneidad, por ser la región de los contactos, las comunicaciones, las facilidades, las oportunidades.

TABLA 2

Categorías lingüísticas: pronunciación, acento, vocabulario

\begin{tabular}{|c|c|c|}
\hline CATEGORÍAS & OPINIONES & PORCENTAJES \\
\hline \multirow[t]{2}{*}{ Pronunciación } & $\begin{array}{l}\text { Mejor pronunciación, gusta más, más } \\
\text { académica, fluida }\end{array}$ & $96,43 \%$ \\
\hline & $\begin{array}{l}\text { No se comen la } / \mathrm{r} / \text { (fuera del Valle } \\
\text { Central) }\end{array}$ & $3,57 \%$ \\
\hline TOTAL & & $100 \%=28$ \\
\hline \multirow[t]{4}{*}{ Acento } & Buen acento, mejor acento & $61,90 \%$ \\
\hline & $\begin{array}{l}\text { Sin acento, con menos acento, acento } \\
\text { neutral }\end{array}$ & $23,80 \%$ \\
\hline & Acento no tan polo, no tan campesino & $9,52 \%$ \\
\hline & Mejor tono & $4,76 \%$ \\
\hline TOTAL & & $100 \%=21$ \\
\hline \multirow[t]{4}{*}{ VOCABULARIO } & $\begin{array}{l}\text { Buen vocabulario, bonito, el mejor } \\
\text { vocabulario, el más amplio }\end{array}$ & $68,75 \%$ \\
\hline & $\begin{array}{l}\text { No cortan las palabras, no se comen las } \\
\text { palabras }\end{array}$ & $12,50 \%$ \\
\hline & $\begin{array}{l}\text { Menos jerga, las palabras no tienen } \\
\text { otros sentidos }\end{array}$ & $12,50 \%$ \\
\hline & Vocabulario menos pachuco & $4,76 \%$ \\
\hline TOTAL & & $100 \%=16$ \\
\hline
\end{tabular}

A continuación, se analizan las tablas anteriores.

Las dos primeras categorías relevantes son sociolingüísticas. La primera categoría por la que se considera que se habla mejor en el Valle Central es por educación y cultura, como lo muestra la Tabla 1 (total: 243 opiniones). Se resalta, por mayoría, que se habla mejor por mayor educación y cultura (o ambiente cultural); luego, se asocia la mejor habla con comportamientos sociales como personas respetuosas, decentes, actualizadas y ecuánimes; es muy frecuente que se asocie la manera de hablar con el comportamiento social y la educación de las personas. Por último, se habla mejor en el Valle Central porque hay más medios o facilidades para educarse.

Seguidamente, la categoría sociolingüística más importante es por corrección y prestigio 
(total: 39 opiniones) de la variedad lingüística del Valle Central. Lo más importante es la comprensión y la claridad de la variedad; en segundo lugar la corrección, el buen uso y la fluidez de la variedad $y$, en tercero, la homogeneidad.

En tercer lugar, la categoría más importante es lingüística. La pronunciación (28 opiniones) es la más importante de las categorías lingüísticas. Se considera que lo cultural y el prestigio, categorías sociolingüísticas, son mucho más importantes que las lingüísticas para valorar positivamente la variedad. Un pequeño porcentaje considera que la pronunciación fuera del Valle Central es mejor porque "no se comen la /r/", opinión relacionada probablemente con el polimorfismo de $/ r /$ en el español vallecentraleño, y se considera que fuera de esa área, la $/ \mathrm{r} /$ se articula de modo vibrante $\mathrm{y}$, por tanto, no se "come".

La siguiente categoría es sociolingüística: se habla mejor por ser del centro del país, de la ciudad capital o, por extensión, del Valle Central. La supremacía de la capital, lo central y las zonas urbanas, industrializadas y más pobladas, le dan un valor agregado a la variedad lingüística de la capital y sus alrededores. La ubicación territorial de la ciudad capital en el centro del país hace también que haya mejores redes de comunicación y más contactos, con lo cual se considera que se "mejora" la variedad lingüística. El hecho de que haya en esta región más oportunidades y facilidades, también es un factor que influye en la mejora de la variedad lingüística (aprox. 21\%).

La quinta categoría es lingüística, y corresponde al acento y al vocabulario. Se considera que la variedad del Valle Central posee el mejor acento y el mejor y más amplio vocabulario. El acento que señalan "no tan polo, no tan campesino", refleja, sin embargo, la inseguridad lingüística de los mismos hablantes del Área Metropolitana, en la que se muestra cierto desdén por la variedad en cuestión; si bien es cierto se considera como habla de ciudad, la más "correcta" y con mejor acento, a su vez se la considera con influencia rural.

Al menos en sus inicios, y como señala Molina Jiménez (2002:90), citando a Bosch
(1963) “[...] San José era la expresión de la democracia rural creada por una sociedad de pequeños propietarios agricultores; [...]". Sin embargo, aun cuando "la cultura urbana de Costa Rica (especialmente la josefina) empezó a europeizarse y a secularizarse con la expansión del café, un proceso que se intensificó en la década de 1840" (Molina Jiménez, 2002:15), y aun con el dinamismo actual de la Gran Área Metropolitana, muchos costarricenses consideran sus orígenes como campesinos. Esto, en cuanto a la lengua se refiere, se percibe en la inseguridad lingüística que ha fomentado la instrucción formal en Costa Rica. Señala Quesada Pacheco (1992:105):

Se hace indispensable señalar que existe en la
actualidad un abismo entre la labor de los lingüistas
en el campo de la lengua materna y las políticas
lingüísticas educativas del estado, las cuales
persiguen objetivos puristas, normativos, donde
muchas características del español de Costa Rica
se continúan tratando como "vicios del lenguaje",
o "incorrecciones", como se enfocaban durante el
siglo XIX, y no como parte de nuestra identidad
idiomático-cultural.

Esta actitud se encuentra aún hoy muy arraigada en la enseñanza de la lengua materna costarricense. En los folletos y cuadernos de enseñanza de la lengua materna se pueden encontrar todavía listas de vocabulario del tipo Appendix probi $^{5}$ en dos columnas que se titulan "no se diga" y "se debe decir", en las que se señalan aspectos tanto de pronunciación y vocabulario, como de gramática.

Señalar que la variedad del Valle Central es mejor porque tiene "menos" acento que las otras variedades de la periferia, demuestra esa inseguridad lingüística, puesto que se sigue considerando con algo de acento, en el sentido de que no es del todo una variedad "sin diferenciación", como es la percepción habitual de un español neutro, libre de acentos y de jergas o de vocabulario característico o distintivo.

En todo lo anterior, subyace una perspectiva conservadora y homogénea de la sociedad costarricense y, en consecuencia, de su variedad de habla. Está presente el aspecto vigilante de la lengua, como lo ha tratado 
acertadamente Jara Murillo (2006:153), en cuyo caso señala una "actitud policial" hacia la lengua en Costa Rica.

\subsection{Actitudes negativas}

¿En cuáles zonas del país considera usted que hablan peor el español?

Un $53 \%$ aproximadamente de los entrevistados indicó que en la periferia del país es donde se habla peor el español, y un $36 \%$ opina que en las zonas marginales y más alejadas de San José es donde se habla peor. En orden de mayor a menor, las regiones específicas en las que peor se habla el español son Limón y Guanacaste, y luego, las zonas marginales de San José. Un 16\% opina que el peor español es el de otras zonas, como Puntarenas, áreas indígenas y rurales. Un porcentaje no despreciable del 13\% es de quienes opinan que en ninguna parte se habla peor el idioma.

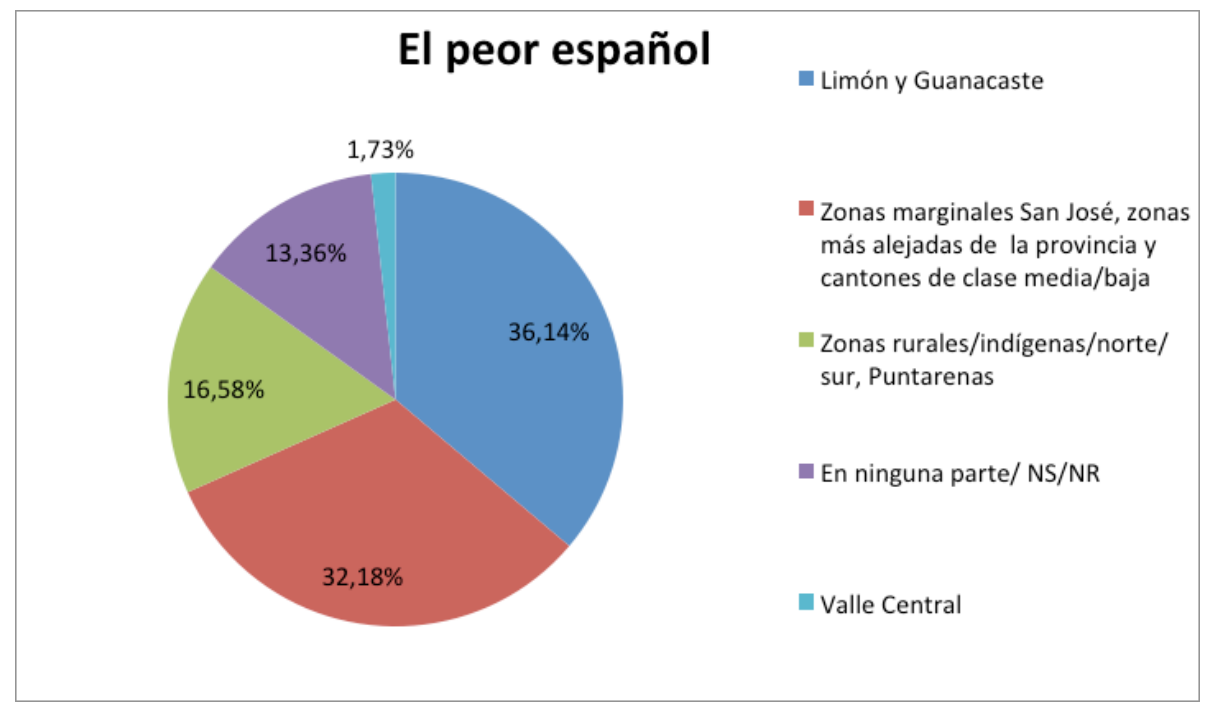

La categoría sociolingüística es la más importante por la que los entrevistados consideran que se habla peor en Costa Rica: lo "raro" lo "otro", lo diferente que se habla, la "mezcla", la diversidad lingüística es considerado lo peor en mayor cantidad. Luego, la incorrección e ininteligibilidad de ciertas variedades "mezcladas" del español, la "impureza"; la diversidad racial, étnica deriva en diversidad lingüística. Para estas personas, la diversidad lingüística y étnica en un país, desprovisten a la lengua de corrección, en especial el habla de los indígenas y de los afrocostarricenses. En estos últimos, ni su español ni su inglés son "correctos"; incluso al inglés de Limón no se le da el estatus de lengua entre la mayoría de los costarricenses.

Como se ha señalado ${ }^{6}$, en nuestra Constitución Política se lee, "El español es el idioma oficial de la Nación. No obstante, el Estado velará por el mantenimiento y cultivo de las lenguas indígenas nacionales"; es decir, no se menciona el inglés criollo como una de las lenguas de Costa Rica. No se incluye, no se le da un nombre, ni siquiera existe... Además, en el Censo de Población del 2011, no se consideró el inglés criollo limonense como una lengua a la cual se le puedan atribuir usuarios, por lo que no se tienen datos oficiales acerca de cuántas personas lo hablan en el país? 
TABLA 3

Categorías sociolingüísticas: incorrección por diversidad étnica/diversidad lingüística, educación y cultura, zonas urbanomarginales y periferia del país

\begin{tabular}{|c|c|c|}
\hline CATEGORÍAS & OPINIONES & PORCENTAJES \\
\hline \multirow[t]{3}{*}{$\begin{array}{l}\text { Incorrección por diversidad étnica/ } \\
\text { diversidad lingüística }\end{array}$} & $\begin{array}{l}\text { Combina, mezclan, revuelven } \\
\text { idiomas o dialectos, hablan otro } \\
\text { dialecto, hablan otra cosa, hablan } \\
\text { raro, hablan indígena, hablan } \\
\text { patuá, hay influencia de otros } \\
\text { idiomas }\end{array}$ & $66,27 \%$ \\
\hline & $\begin{array}{l}\text { Hablan un mal español, vulgar, } \\
\text { incorrecto, no es puro, no conocen } \\
\text { bien el idioma }\end{array}$ & $30,12 \%$ \\
\hline & $\begin{array}{l}\text { Están revueltos con negros, por } \\
\text { ser negros }\end{array}$ & $3,61 \%$ \\
\hline TOTAL & & $100 \%=83$ \\
\hline \multirow[t]{3}{*}{$\begin{array}{l}\text { Educación } \\
\text { y cultura }\end{array}$} & $\begin{array}{l}\text { Educación baja, diferente, falta } \\
\text { de estudios }\end{array}$ & $52 \%$ \\
\hline & $\begin{array}{l}\text { Chusma, malas costumbres, } \\
\text { pachucos, mañosos, vagos para } \\
\text { hablar, mal hablados }\end{array}$ & $27,87 \%$ \\
\hline & $\begin{array}{l}\text { Bajo nivel cultural, por diversidad } \\
\text { cultural }\end{array}$ & $19,67 \%$ \\
\hline TOTAL & & $100 \%=63$ \\
\hline \multirow[t]{2}{*}{$\begin{array}{l}\text { Zonas urbanomarginales y periferia del } \\
\text { país }\end{array}$} & $\begin{array}{l}\text { Zonas marginales, barrios bajos, } \\
\text { pobreza, bajos recursos }\end{array}$ & $68 \%$ \\
\hline & $\begin{array}{l}\text { Habla rural, de pueblo, de campo, } \\
\text { incivilizados }\end{array}$ & $32 \%$ \\
\hline TOTAL & & $100 \%=25$ \\
\hline
\end{tabular}

Revisando este tipo de actitudes y creencias, se coincide igualmente con van Dijk (1980:41) en que "las actitudes son sistemas 'complejos' porque organizan varias clases de información cognitiva. Por lo tanto, una actitud, por ejemplo, acerca de los 'negros' puede contener creencias, ("Los negros son holgazanes, musicales," etc.), opiniones (Los negros deberían permanecer separados de los blancos"), y conocimientos ("Los negros viven en ghettos, son pobres", etc.)."

En la opinión del autor, las actitudes, junto con mucha otra información son la energía para la formación de posibles acciones, y no son solamente una predisposición hacia estas. Y agrega que "precisamente como valores generales, también las normas pueden pertenecer al sistema de actitudes (por ejemplo: "Es bueno segregar a los negros; no es bueno crear especial bienestar social o programas de universidad para ellos", etc.).

En esta misma línea, se podría señalar que el sistema de actitudes anteriormente descrito por los hablantes del español de San José abarca, en cierta forma, una "norma" compartida en este tipo de sociedad en la que lo diferente, lo que no es homogéneo es lo peor (actitud negativa), y que precisamente por ser negros (o indígenas) estos hablantes no pueden expresarse de otra manera que no sea mal. Así, 
la actitud lingüística también promueve en forma clara la discriminación racial, con sus consecuentes acciones.

La segunda categoría es más frecuente por la que se habla peor el español es lingüística, $y$ se trata del acento (probablemente rasgos suprasegmentales, con 83 opiniones). Para estos hablantes, el peor acento está en la periferia del país, y se considera diferente, raro, feo, cantado, se considera "otro" acento. En ese sentido, en el conjunto de rasgos que se considera "acento" no se acepta la diversidad y esta es valorada muy negativamente. Por otro lado, opinan que se habla peor porque "hablan como nicas", tratándose principalmente de las referencias al habla de Guanacaste ${ }^{8}$ y de las regiones urbanomarginales del Valle Central; por influencia del "país vecino/cercano", el cual no se nombra, pero se supone que no se trata de Panamá; el acento de "inmigrantes" es subvalorado también, al igual que el de los "extranjeros", $\sin$ especificar la nacionalidad. Sin embargo, también se sobreentiende que se trata del habla nicaragüense. A este respecto, se estima que se trata de una actitud histórica el considerar como inferior la cultura nicaragüense y, más en general, la centroamericana. Señala Molina Jiménez, (2002:8):

La identidad nacional, que se configuró a partir de la década de 1880, se caracterizaba por su extroversión cultural (dada su orientación europeizante), su desprecio por lo centroamericano, y su énfasis por la excepcionalidad de Costa Rica, la cual tenía, según los políticos e intelectuales liberales, una decisiva base étnica. La visión oficial destacaba que el país, en contraste con sus vecinos del istmo, y sobre todo con Nicaragua, era una república blanca - una feliz Suiza de los trópicos-, cuyos gobiernos civiles y democráticos se afanaban por ofrecer a la población crecientes servicios educativos y sanitarios con el fin de garantizar la pureza y el progreso de una raza casi perfecta.

Se observan hasta aquí con toda claridad, las actitudes de discriminación étnica y de xenofobia de muchos de los josefinos a través de las valoraciones acerca del habla Esto corresponde a la discriminación que ha originado el fenómeno de la inmigración y la xenofobia propia del "país vecino".

TABLA 4

Categoría lingüística: acento, vocabulario, pronunciación y gramática

\begin{tabular}{lll}
\hline CATEGORÍAS & OPINIONES & PORCENTAJES \\
\hline Acento & $\begin{array}{l}\text { Acento raro, diferente, feo, malo, cantado, tienen otro } \\
\text { acento }\end{array}$ & $75,39 \%$ \\
\hline & $\begin{array}{l}\text { Acento nica, del país cercano, del país vecino, de } \\
\text { inmigrantes, de extranjero }\end{array}$ & $24,61 \%$ \\
\hline TOTAL & & $\mathbf{1 0 0} \%=\mathbf{6 5}$ \\
\hline Vocabulario & Vocabulario malo, pobre, inadecuado, usan palabrotas & $52 \%$ \\
\hline & Cambian las palabras, usan palabras diferentes & $32 \%$ \\
\hline TOTAL & Usan apodos, dichos, jergas & $16 \%$ \\
\hline Pronunciación & Mala pronunciación & $\mathbf{1 0 0} \%=\mathbf{2 5}$ \\
\hline & Cambian la pronunciación & $61,54 \%$ \\
\hline TOTAL & Se comen letras, se comen vocales & $23,08 \%$ \\
\hline Gramática & No conjugan verbos & $15,38 \%$ \\
\hline & No terminan frases & $\mathbf{1 0 0} \%=\mathbf{1 3}$ \\
\hline Usan frases diferentes & $50 \%$ \\
\hline & & $25 \%$ \\
\hline
\end{tabular}


La tercera categoría en importancia es sociolingüística (educación y cultura $=63$ valoraciones). Se señala que las personas hablan mal porque les falta educación formal, o porque tienen estudios "diferentes" (podrían referirse a los extranjeros inmigrantes). Además, hablan mal las personas de malas costumbres, la "chusma", los malhablados, los "vagos" (vagabundos) para hablar y las personas "mañosas". Es frecuente que se correspondan hábitos de comportamiento social con hábitos lingüísticos. Además, algunos consideran que la poca cultura o la diversidad cultural son responsables de que los hablantes posean un habla deficitaria. De nuevo, lo diferente es lo peor en el habla.

La cuarta categoría en importancia es sociolingüística, y se relaciona con las zonas urbanomarginales y periferia del país (25 opiniones). Las personas que están fuera del centro del país son las que hablan peor. Las zonas urbanomarginales son peor valoradas en su habla que las rurales, a las cuales también llaman "de campo", "de pueblo" o "incivilizadas".

Por último, se considera que lo que menos incide en que las personas hablen peor es la categoría lingüística, con excepción del acento. Primero, por vocabulario (25 valoraciones), tienen vocabulario pobre, inadecuado, "malo", el uso de "palabrotas"; siempre el cambio y la diferencia se valoran negativamente (cambian las palabras o usan palabras diferentes), y por apodos, dichos y jergas; luego por pronunciación (13 valoraciones), pronunciación "mala" o "diferente" $\mathrm{y}$, por último, por gramática: no conjugan verbos, no terminan frases (solo 4 valoraciones).

\section{Conclusiones}

Como era de esperar en el segmento de población estudiado, el cantón central de San José, las actitudes positivas se enfocan hacia el Valle Central y, especialmente, hacia la ciudad capital como centro de irradiación cultural, educativo y lingüístico. Se tiene la homogeneidad lingüística propia de esa zona como lo prestigioso, "correcto", general y "normal ${ }^{10 "}$.
Solo en un tercer plano se exhiben criterios lingüísticos por los cuales el habla del Valle central es mejor: pronunciación, acento y vocabulario en un plano mucho menor. Se percibió un poco de inseguridad lingüística respecto del acento, pues se considera aún algo campesino y se percibe todavía con acento diferenciador. Es decir, no es aún lo suficientemente homogéneo, invariable y general como para considerarse un acento no diferenciador.

Las actitudes negativas muestran resultados algo inquietantes pero poco sorprendentes en el contexto de una sociedad como la costarricense, dada a la históricamente determinada discriminación étnica y a la xenofobia, como lo corroboran los textos de Molina (1986 y 2002). Se juzgan negativamente la heterogeneidad y la variación lingüística; se observan, como fuente de desigualdad lingüística y sociocultural, actitudes de discriminación étnica contra los afrocostarricenses e indígenas y contra sus lenguas. Incluso la incorrección del español se le atribuye también a este grupo.

De las categorías lingüísticas, la más importante es, en este caso, el acento. Tienen mal acento principalmente los guanacastecos $\mathrm{y}$, en general, quienes viven en la periferia del Valle Central (zonas urbanomarginales) y en la periferia del país. El acento guanacasteco se atribuye a la (mala) influencia del español nicaragüense. Se observan entonces actitudes xenofóbicas en los hablantes y una actitud de desigualdad reflejada también en la economía, pues las clases más desposeídas son las que se considera que hablan peor y con influencia, a su vez, de las hablas de los desposeídos e inmigrantes nicaragüenses.

El factor de la educación siempre muestra relevancia tanto para hablar bien (alta escolaridad) como hablar mal (baja escolaridad). Las diferencias restantes, por pronunciación, vocabulario o sintaxis, son reprobadas cuando se habla de la periferia del país. De nuevo, lo diferente es valorado de forma negativa y reproduce un sistema de actitudes fundamentado en una ideología de la sociedad costarricense que se considera aquí conservadora, homogeneizante y discriminadora de la diversidad. 


\section{Nota final}

A la luz del coloquio de la Academia Costarricense de la Lengua Española, en su nonagésimo aniversario el pasado año, "Las academias de la lengua española y su proyección en la sociedad", es algo alentador percibir nuevos aires en la otrora acartonada y retrógrada actividad de las Academias, lideradas por la Real Academia Española de la Lengua Española, cuyo propósito inicial fue el de:

[...] «fijar las voces y vocablos de la lengua castellana en su mayor propiedad, elegancia y pureza». Se representó tal finalidad con un emblema formado por un crisol en el fuego con la leyenda Limpia, fija y da esplendor, obediente al propósito enunciado de combatir cuanto alterara la elegancia y pureza del idioma, y de fijarlo en el estado de plenitud alcanzado en el siglo XVI. Tomado de http://www.rae.es/rae/ gestores/gespub000001.nsf/voTodosporId/CEDF300 E8D943D3FC12571360037CC94

En la actualidad, los académicos han percibido que los usos no se pueden prescribir como se prescribe un medicamento para combatir una enfermedad; es decir, que la norma no se impone, y que hay muchas normas y todas son igualmente válidas. Las Academias son ahora más descriptivas que prescriptivas $\mathrm{y}$, como lo ha afirmado José Luis Vega, director de la Academia Puertorriqueña de la Lengua: "Las academias no sirven para decir a la gente cómo debe hablar ${ }^{11 " . . ~ E n ~ e l ~ m i s m o ~ s e n t i d o, ~}$ otros académicos han aclarado la función actual de las Academias:

[...] el chileno, Alfredo Matus Olivier: "Las academias no sirven para condenar, censurar, estigmatizar, prohibir, discriminar, dominar. No son los verbos que conjugan". "Las Academias no son 'policías de la lengua'; más bien, se asemejan a los notarios pues dan fe de lo que ocurre", agrega el director peruano, Marco Martos Carrera.

"Las academias no sirven para poner un cinturón de castidad (o de casticidad) al idioma", sostiene Daniel R. Fernández, miembro de la directiva de la Academia Norteamericana de la Lengua.

¿Para qué sí? Entonces, ¿para qué sirven las academias? Mario Antonio Sandoval Samayoa, director de la Academia Guatemalteca, opina: "La academias señalan la corrección lingüística, lo que no significa necesariamente el estancamiento lingüístico. Las academias deben cuidar esos cambios para que sean avances". Tomado de http:// www.nacion.com/ocio/artes/Coloquio-reunedirectivos-academias-lengua_0_1357864225.html.

Y se agrega:

Todos hacemos el idioma, pero ¿hay un idioma español correcto? "No exactamente. La corrección lingüística es una utopía. Hay muchos idiomas españoles correctos: tantas patrias de corrección lingüística (o de incorrección) como existan necesidades y empeños por dominar (o no) la lengua", opina el director venezolano.

José Manuel Blecua añade: "No existe un español más 'correcto' que otro; no hay un modelo único. Nuestra lengua común, con todas sus variantes $\mathrm{y}$ con todos sus acentos, es un gran patrimonio que debemos preservar por encima de localismos limitadores". Tomado de http://www.nacion.com/ ocio/artes/Coloquio-reune-directivos-academiaslengua_0_1357864225.html.

Con estas citas de los académicos se corrobora que hay un giro no solo de punto de vista, sino de perspectiva, de interpretación de la norma y del concepto de corrección en la lengua. Se espera que estos cambios lleguen paulatinamente a la población, y puedan disminuirse la inseguridad y los estereotipos lingüísticos, los cuales solo han contribuido a aumentar los prejuicios, la desigualdad y la discriminación en muchos sentidos.

\section{Notas}

1. Calvo y Castillo (2013). Proyecto LIAS, Universidad de Bergen (en prensa).

2. En la Nota 1 del artículo del 2010 se indica: "El presente artículo apareció por primera vez en la Revista de Filología y Lingüistica de la Universidad de Costa Rica, Vol. XII, No. 2, 1 (págs. 113128). En esa publicación se suprimió por error una parte fundamental de la metodología, lo que significó serios problemas para la comprensión de la investigación."

3. L'Institut Universitari de Lingüística Aplicada (IULA) és un centre de recerca i formació de 
postgrau de la Universitat Pompeu Fabra que aplega un nombrós conjunt d'investigadors, col-laboradors i becaris. En http://www.iula.upf.edu/presca.htm Consultado en agosto de 2011.

4. Tomado de González Martínez (2008).

5.

Cfr. http://sun.iwu.edu/ cisabell/courses/ spanish403/handouts/appendix_probi.pdf

6.

Al respecto, ver Hernández Sánchez (2013): http:/ www.nacion.com/ocio/artes/Limon-literaturagastronomia-Costa-Rica_0_1336866330.html

7. Al respecto, ver Meléndez Obando (2001): http:// wvw.elfinancierocr.com/ef_archivo/2001/enero/28/ estilos1.html

8. Señala la historiadora Sylvia Muñoz (2009): [...] el Partido de Nicoya, que hoy parte de la provincia de Guanacaste... tuvo, durante la época de la colonia española, varios estatus administrativos. Perteneció a Nicaragua, luego se mantuvo independiente, posteriormente se le unió a Costa Rica y así se intercambiaron el Partido hasta 1824, cuando finalmente nuestro país vio aumentado su territorio por la unión de Nicoya. Consideramos que es importante explicar por qué a Nicoya se le llamaba Partido; según la Constitución de Cádiz, se consideraba Partido a una división administrativa con cierta autonomía, que hoy se asemeja, dentro de la perspectiva del derecho administrativo, a un cantón. El Partido de Nicoya era, en ese tanto, un cantón que no pertenecía, al momento de su anexión, a ninguna provincia.

Cf. Solís (2009:92). El autor señala: "En el caso de Costa Rica, la xenofobia puede explicarse psicológicamente, por medio de lo que Freud denominó narcisismo por la mínima diferencia; económicamente, por la situación económica de la región y la percepción que tienen los costarricenses de que los extranjeros vienen a explotar sus recursos, saturar los servicios públicos y acaparar los empleos disponibles; por razones de contacto, debido a la superioridad que tienden a manifestar los costarricenses en el trato cotidiano con los extranjeros; e históricamente, por cuanto Costa Rica se ha caracterizado por un imaginario etnocéntrico que ha acompañado su formación como Estado nación independiente.

10. Cf. Lope Blanch (1993) y http://congresosdelalengua. es/valladolid/ponencias/unidad_diversidad_del_ espanol/1_la_norma_hispanica/lope_j.htm
11. Tomado de: http://www.nacion.com/ocio/ artes/Coloquio-reune-directivos-academiaslengua_0_1357864225.html

\section{Bibliografía}

Antología del latín vulgar: Appendix Probi. http://sun.iwu.edu/ cisabell/courses/ spanish403/handouts/appendix_probi.pdf Consultado en agosto de 2013.

Arrieta, M., C. Jara y C. Pendones. (1986). Actitudes lingüísticas hacia dos variedades de habla: Valle Central y Guanacaste. Revista de Filología y Lingüistica de la Universidad de Costa Rica, Vol. XII, No. 2, (págs. 113-128).

. (2010). Actitudes lingüísticas hacia dos variedades de habla: Valle Central y Guanacaste. Káñina, Rev. Artes y Letras, Univ. Costa Rica. XXXIV (1): 115-127.

Calvo-Shadid, Annette y Jacqueline Castillo Rivas. (2012). Las actitudes lingüísticas en el español de San José, Costa Rica. (en prensa)

Drevdal, S. (2009). Actitudes lingüísticas de los inmigrantes nicaragüenses en Costa Rica hacia su propia habla. Tesis de maestría. Universidad de Bergen.

González-Martínez, J. (2008). Metodología para el estudio de las actitudes lingüísticas. En Actas del XXXVII Simposio Internacional de la Sociedad Española de Lingüistica (SEL). Pamplona, Servicio de Publicaciones de la Universidad de Navarra, 2008:229-238.

Hernández-Sánchez, M. (2013). http://www. nacion.com/ocio/artes/Limon-literaturagastronomia-Costa-Rica_0_1336866330. html Consultado en agosto de 2013. 
Jaén-Garcia, X. (1991). Las actitudes lingüísticas de los hablantes de Sardinal, Carrillo, Guanacaste, hacia su propia habla. Tesis de maestría. Universidad de Costa Rica.

Jara-Murillo, Carla Victoria. (2006). El español de Costa Rica según los ticos. Un estudio de lingüistica popular. San José: Editorial de la Universidad de Costa Rica.

La Nación: http://www.nacion.com/ocio/artes/ Coloquio-reune-directivos-academiaslengua_0_1357864225.html Consultado en agosto de 2013.

Lope-Blanch, Juan Miguel. (1993). Nuevos estudios de Lingüistica hispánica. México, D.F.: Universidad Nacional Autónoma de México.

. (2001). La norma lingüística hispánica. II Congreso Internacional de la Lengua Española, «El español en la sociedad de la información».

López-Morales, H. (2004). Sociolingüística. Madrid: Gredos.

Meléndez Obando. (2001). http://wvw. elfinancierocr.com/ef_archivo/2001/ enero/28/estilos1.html Consultado en agosto de 2013.

Molina-Jiménez, Iván. (1986). El Valle Central de Costa Rica en la independencia. En Revista de Historia. San José, № 14 (julio-diciembre, 1986), pp. 85-114.

(2002). Costarricense por dicha. Identidad nacional y cambio cultural en Costa Rica durante los siglos XIX y $X X$. Ciudad Universitaria Rodrigo Facio: Editorial Universidad de Costa Rica.

Muñoz, Sylvia. (2009). La anexión del Partido de Nicoya. En http://www.tiquicia.com/ articulos/cultura/anexion.asp Consultado en abril de 2013.

Quesada-Pacheco, Miguel Ángel. (1992). El español en Costa Rica. Historia de sus estudios filológicos y lingüísticos. San José: Editorial Fernández Arce.

. (2005). Papel del español americano en la enseñanza del ELE. FIAPE: Primer congreso internacional: El español, lengua del futuro. Toledo: págs. 20-23. Consultado en: http://www.educacion.gob. es/redele/biblioteca2005/fiape/quesada. pdf Consultado en marzo del 2011.

. (2008). De la norma monocéntrica a la norma policéntrica en español. Algunas reflexiones históricas según testimonios y actitudes lingüísticas. ANPE: II Congreso nacional: multiculturalidad y norma policéntrica: Aplicaciones en el aula de ELE. (págs. 1-36) En: http://www. educacion.gob.es/redele/Biblioteca2009/ anpe/ANPE2008MiguelAngelQuesada. pdf Consultado en mayo de 2011.

Real Academia Española de la Lengua http:// www.rae.es/rae/gestores/gespub000001. nsf/voTodosporId/CEDF300E8D943D3FC 12571360037CC94 Consultado en agosto de 2013.

Solís-Obiols, Marina. (2002). L'aparellament de veus disfressades: una prova clàssica en el mesurament formal de les actituds lingüístiques. Noves SL. Revista de Sociolingüistica 1. http://www6. gencat.net/llengcat/noves/hm02estiu/ metodologia/solis1_5.htm\#2 Consultado en setiembre de 2013 .

Solano, Y. y Umaña, J. (1994). Inseguridad lingüística del universitario costarricense. En Revista de Filología y Lingüística de la Universidad de Costa Rica. Vol.XX, No 1 (págs.168-178). 
Solís, Pedro. (2009). El fenómeno de la xenofobia en Costa Rica desde una perspectiva histórica. En Revista de Filosofía de la Universidad de Costa Rica. Volumen 28, Número 120-121, enero-agosto (págs. 91-97).

Umaña-Aguiar, J. (1989). La relación entre actitudes lingüísticas conducta e identidad. Revista de Filología y Lingüistica de la Universidad de Costa Rica, Vol. XV, No. 2 (págs. 121- 129).

.(1990). Grupos portadores de actitudes lingüísticas. Revista de Filología y Lingüistica de la Universidad de Costa Rica, Vol. XVI, No. 2, (págs. 103- 109).
Universitat Pompeu Fabra: IULA (Institut Universitari de Lingüística Aplicada. http://www.iula.upf.edu/uval/upres2es. htm Consultado en agosto de 2011.

van Dijk, Teun A. (1980). Algunas notas sobre la ideología y la teoría del discurso. En Semiosis. (Universidad Veracruzana, Xalapa, México), $\mathrm{n}^{\mathrm{o}}$ 5, julio-diciembre de 1980, pp. 37-53.

. (1996). Análisis del discurso ideológico. En Semiosis. (Universidad Veracruzana, Xalapa, México), $\mathrm{n}^{\circ}$ 5, julio-diciembre de 1980, pp. 37-53. 
